\title{
Evidence for viable and stable triploid Trypanosoma congolense parasites
}

\author{
Eliane Tihon, Hideo Imamura, Jean-Claude Dujardin and Jan Van Den Abbeele ${ }^{*}$
}

\begin{abstract}
Background: Recent whole genome sequencing (WGS) analysis identified a viable triploid strain of Trypanosoma congolense. This triploid strain BANANCL2 was a clone of the field isolate BANAN/83/CRTRA/64 that was collected from cattle in Burkina Faso in 1983.

Results: We demonstrated the viability and stability of triploidy throughout the complete life-cycle of the parasite by infecting tsetse flies with the triploid clone BANANCL2. Proboscis-positive tsetse flies efficiently transmitted the parasites to mice resulting in systemic infections. WGS of the parasites was performed at all life-cycle stages, and a method based on a block alternative allele frequency spectrum was developed to efficiently detect the ploidy profiles of samples with low read depth. This approach confirmed the triploid profile of parasites throughout their life-cycle in the tsetse fly and the mammalian host, demonstrating that triploidy is present at all stages and is stable over time.

Conclusion: The presence of viable field-isolated triploid parasites indicates another possible layer of genetic diversity in natural T. congolense populations. The comparison between triploid and diploid parasites provides a unique model system to study the impact of chromosome copy number variations in African trypanosomes. In addition, the consequences of triploidy can be further investigated using this stable triploid model.
\end{abstract}

Keywords: Trypanosoma congolense, Triploidy, Whole genome sequencing

\section{Background}

African trypanosome genomes are considered to be diploid [1, 2]. Yet, triploid T. brucei parasites have been observed following experimental crossing [3-5]. It has been postulated that triploid T. brucei parasites are atypical but not lethal [6], and that they resulted from either the fusion between diploid and haploid nuclei, or from failures during meiosis due to parental strains possessing very divergent homologous chromosomes $[5,7]$.

Meiosis in African trypanosomes is a complex process so far only described for T. brucei. Non-obligatory mating of T. brucei [5] occurs in the tsetse fly salivary glands where haploid gametes can be found and meiosisspecific genes are expressed [5, 8-10]. This is the lifecycle stage at which triploid $T$. brucei potentially emerge. However, no triploid strains of $T$. brucei have been reported from the field, suggesting that viable triploids are rare or have been frequently overlooked.

\footnotetext{
* Correspondence: jvdabbeele@itg.be

Department of Biomedical Sciences, Institute of Tropical Medicine, Nationalestraat 155, 2000 Antwerp, Belgium
}

In a previous phylogenomic study of $T$. congolense, we discovered a triploid strain isolated from infected cattle in Burkina Faso which represented the first documented case of triploidy in a field-isolated African trypanosome [11]. There is currently no further information about its frequency in natural trypanosome populations or its persistence and impact on its viability throughout its lifecycle. Moreover, to understand the underlying mechanisms of triploidy emergence in $T$. congolense, further knowledge about the existence of a putative sexual stage during the parasite life-cycle is required.

In the T. congolense life-cycle, the presence of a sexual stage with the expression of meiosis-specific genes has currently not been formally identified. However, recent genetic studies on T. congolense field populations clearly indicated the existence of recombination between different parasite populations, suggesting the existence of a sexual stage in the species life-cycle [11].

In this study, we monitored the ploidy status of the $T$. congolense clone BANANCL2 during its development in the tsetse fly and the mammalian host following an 
infected tsetse bite. We found that triploidy is a stable trait that persists throughout the parasite life-cycle; the triploid parasites are viable at all stages and fully capable of infecting the murine host when they were inoculated through tsetse fly bite.

\section{Methods}

Origin of the samples and parasite isolation from mouse blood

The $T$. congolense savannah strain BANAN/83/CRTRA/ 64 was collected in 1983 in Burkina Faso [12]. The clones BANANCL1, BANANCL2 and BANANCL3 were obtained after infecting rodents with a single parasite of the BANAN/83/CRTRA/64 strain using the micro-drop method [13]. The savannah strain KTT/MSOROM7C1 (referred to as MSOROM7 in this study) was isolated in Zambia in 2003 [13]. The savannah strain MBOI/BK/89/ SA268 (referred to as SA268 in this study) was isolated in Burkina Faso in 1989 [14]. MSOROM7 was selected because of its high depth coverage (i.e. over $84 \times$ ) which was suitable for computational simulation for mixing diploidy and triploidy samples. SA268 was selected because it was genetically very similar to BANANCL1. All parasite strains were morphologically indistinguishable.

The $T$. congolense strains were maintained in OF-1 mice. The isolation of parasites from mouse blood was performed as described previously [11].

\section{Tsetse flies infections and parasite extraction}

Glossina morsitans morsitans were obtained from a colony at the Institute of Tropical Medicine, Antwerp, Belgium. Their origin and rearing conditions are described elsewhere [15].

Tsetse fly blood meals were prepared using mouse blood infected with T. congolense BANANCL2. This was diluted to a final concentration of $2.5 \times 10^{6} \mathrm{tryp} / \mathrm{ml}$ in $15 \mathrm{ml}$ defibrinated horse blood and supplemented with $10 \mathrm{mM}$ reduced L-glutathione. Freshly emerged tsetse flies were then fed $24 \mathrm{~h}$ after emergence. Afterwards, flies were maintained for 28 days by feeding them 3 times a week on clean defibrinated horse blood. At the end of the experiment, the midgut, proventriculus and proboscis of each fly was dissected and parasites were collected for DNA extractions. Three independent infection experiments were performed. Before dissection, some proboscis-infected tsetse flies were used to probe on anaesthetized mice, resulting in systemic blood infections [16]. Parasites from these infected mice were collected for DNA extraction as described previously [11].

DNA extraction, high-throughput sequencing, read mapping and variant calling

DNA extractions were performed using the QIAamp DNA Blood Mini Kit (Qiagen, Hilden, Germany) according to manufacturer's instructions. Whole genome sequencing (WGS) experiment and analyses were performed as mentioned previously [11].

\section{Estimating allele frequency profile from SNP blocks}

In the WGS, the DNA strands are sequenced randomly and a high depth coverage will ensure that each DNA strand (and their corresponding nucleotide sequences) is well represented in the analysis. In this case the alternative allele frequency is calculated as the ratio of read depth of an alternative allele over the total read depth at heterozygous SNP sites. This is similar to the method described in [17]. However, the accurate estimation of the alternative allele frequency distribution of a sample with a read depth coverage lower than $30 \times$ is hampered because the proportion of each DNA strand may not be equally represented in the sequence analysis, resulting in an uneven SNP distribution for the SNP variants. This problem is more pronounced in a triploid sample because of the presence of an additional DNA strand. Normally, triploid samples with high read depth show two peaks at 0.33 and 0.66 in their alternative allele frequency distribution [17]. Due to larger uncertainty for allele frequency estimation for low read depth samples, these two peaks start to overlap, making it impossible to distinguish their ploidy status unambiguously.

Therefore, to overcome this problem we developed an alternative allele frequency calculation method based on blocks of 1000 SNP sites. Here, both heterozygous and homozygous SNP sites were included since they could not be properly distinguished at low depth sites. This method aggregates the read depth information of multiple read positions (i.e. 1000 SNP sites) for better allele frequency estimation. For all of the 1000 individual SNP sites of each block, we summed up separately the frequency of alleles corresponding to the reference bases (Ref) and the frequency of alleles corresponding to the alternative bases (Alt). The alternative allele frequency of each block was then estimated using the formula Alt / $($ Ref + Alt). This provided an aggregate alternative allele frequency for a given block (i.e. the block alternative allele frequency) that reflected the proportion of homozygous and heterozygous sites found within the block (Additional file 1: Figure S1). For instance, a block alternative allele frequency close to 1 suggests that most of the 1000 SNP sites within the block are homozygous. Likewise, a block alternative allele frequency of 0.8 suggests a combination of mostly homozygous SNP sites and few heterozygous SNP sites. Other frequency values could reflect a combination of multiple SNPs of different heterozygosity. For instance, in a triploid sample, a frequency of 0.5 could represent a block entirely heterozygous with $50 \%$ of the SNPs with a frequency of 0.33 and $50 \%$ of the SNPs with a frequency of 0.66 ; or could 
reflect a combination of heterozygous and homozygous SNP sites (Additional file 1: Figure S1). We combined the block alternative allele frequencies and obtained a profile of aggregate alternative allele frequencies for all the chromosomes.

We performed the block alternative allele frequency calculation for BANAN/83/CRTRA/64, BANANCL1, BANANCL2, BANANCL3, the 16 BANANCL2 samples from fly tissues and tsetse-infected mouse blood, and SA268. In our analysis, BANANCL2, which had the highest read depth and provided the most reliable allele frequency estimation, was set as standard triploid sample, and SA268 was set as standard diploid sample. It should be noted that these profiles are strain-specific and could only be used to determine the ploidy profiles of genetically similar strains. In addition, we calibrated the block alternative allele frequency method for various depths using different fractions of the BANANCL2 sample. We created the block alternative allele frequency profiles of a depth range of $79 \times, 44.5 \times, 22.3 \times, 13.4 \times$, $10.4 \times, 5.3 \times, 1.15 \times, 0.58 \times, 0.27 \times$ to $0.1 \times$ (i.e. using 50 $0.1 \%$ of the sample depth coverage). The resulting allele frequency profiles were consistent with those obtained with a sufficient depth coverage, indicating the accuracy of this technique to estimate the allele frequency profiles for samples with lower depth coverage. In addition, we saw that the profile of BANAN-PB-2 (i.e. lowest depth coverage of the dataset), corresponded to the profiles of BANANCL2 fractions with depths between 1.15 and 0.27 (data not shown).

\section{Flow cytometry}

The DNA contents of BANANCL2 and MSOROM7 (i.e. diploid strain) were measured by flow cytometry using the propidium iodide flow cytometry kit (Abcam; $\lambda_{\text {ex }}$ : $493 \mathrm{~nm} ; \lambda_{\mathrm{em}}: 636 \mathrm{~nm}$ ) according to the manufacturer's instructions. Briefly, parasites were DEAE-purified from OF-1 mice blood. For each condition, $10^{7}$ trypanosomes were fixed and permeabilized overnight with $70 \%$ icecold methanol. The parasites were washed twice with Phosphate Buffer Saline (PBS), and stained with a solution containing $50 \mu \mathrm{g} / \mathrm{ml}$ propidium iodide and $550 \mathrm{U} /$ $\mathrm{ml}$ ribonuclease $\mathrm{A}$. The parasites were incubated for 30 min at RT in the dark and were analyzed with a BD FACSVerse flow cytometer and the BD FACSuite software v1.0.3.

\section{Results}

Predominance of the triploid population in a mixed $T$. congolense infection

We previously discovered a triploid $T$. congolense strain that showed a hybrid profile between parasites from both Zambia and Burkina Faso [11]. This sample, BANANCL2, was a clone of the BANAN/83/CRTRA/64 isolate that was collected from cattle in 1983 in Burkina Faso [12]. The triploid status of the BANANCL2 clone was identified by measuring the alternative allele frequencies at heterozygous sites based on WGS data [11]. In a diploid strain, the alternative allele frequency distribution is bell shaped with its peak around 0.5 for each chromosome, as observed in the $T$. congolense diploid strains SA268 and MSOROM7 (Fig. 1a). In contrast, the allele frequency distribution of BANANCL2 is completely different with two distinct peaks at around 0.33 and 0.66 for each chromosome which is characteristic of triploidy (Fig. 1a) [17].

We further confirmed the triploid status of the BANANCL2 clone by quantifying and comparing its DNA content to that of the diploid MSOROM7 strain by flow cytometry (Fig. 2). The DNA content was measured on fixed and permeabilized cells stained with propidium iodide as described in [5]. In the diploid MSOROM7, the first major peak (a) corresponded to $2 \mathrm{~N}$ DNA cells in the G1/G0 cell cycle stage, and the second peak (c) to cells with a 4 N DNA content (i.e. G2 stage prior to cell division). For the BANANCL2 clone, the major peak (b) corresponded to cells with a $3 \mathrm{~N}$ DNA-content (i.e. between the $2 \mathrm{~N}$ and $4 \mathrm{~N}$ peaks of MSOROM7 cells) whereas the second peak (d) reflected a $>4$ N DNA-content. This result confirmed the triploid status of BANANCL2 that was observed by WGS analysis.

To assess whether the triploid status of BANANCL2 was a stable trait already present in the original field sample, or whether it was acquired during the cloning procedure, we sequenced the whole genome of the original BANAN/83/CRTRA/64 field isolate and its other derived clones BANANCL1 and BANANCL3, and estimated their alternative allele frequency profiles. The alternative allele frequency spectra of both BANAN/83/ CRTRA/64 and BANANCL3 showed a clear peak at 0.33 and a second less-pronounced peak at 0.66 , while the alternative allele frequency profile of BANANCL1 did not highlight specific profile due to its low depth coverage (Fig. 1b, Table 1).

For the calculation of allele frequencies from samples with average depth coverage lower than $30 \times$, we estimated the alternative allele frequencies based on blocks of 1000 SNP sites instead of on individual heterozygous SNP sites. We then obtained an average alternative allele frequency for a given SNP block (see Methods). We first determined the profile of the block alternative allele frequencies of the clone BANANCL2 and the strain SA68 to establish the reference base line profile of a triploid and diploid parasite population, respectively (Fig. 3a, b; Additional file 1: Figure S1). Using this approach, we identified that BANAN/83/CRTRA/64 and BANANCL3 were triploid, as characterized by a block alternative 


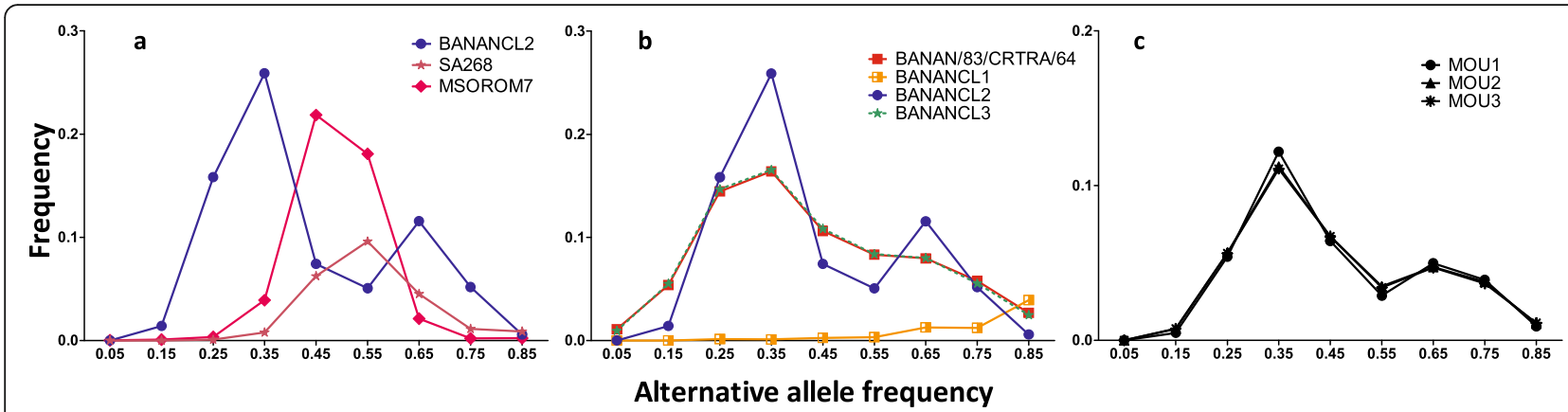

Fig. 1 Alternative allele frequency profiles based on 11 chromosomes. The alternate allele frequencies are shown for: a the diploid strains SA268 and MSOROM7 and the triploid clone BANANCL2; b BANAN/83/CRTRA/64 parasites and its clones BANANCL1, BANANCL2 and BANANCL3; $\mathbf{c}$ the BANANCL2 parasites extracted from the blood of tsetse-bite infected mice after completion of the full parasite cycle in the tsetse vector. The distribution of read depths for alternative alleles at heterozygous sites showed two peaks at around 0.33 and 0.66 for triploid chromosomes, and one peak at around 0.5 for diploid chromosomes. Note, these alternative allele frequencies were calculated based on each heterozygous SNP site for all chromosomes

allele frequency profile similar to BANANCL2 (Fig. 3a) (i.e. correlation values of $r^{2}=0.996$ and $r^{2}=0.997$, respectively; Table 2). In contrast, BANANCL1 had a block alternative allele frequency profile similar to SA268 and was therefore classified as diploid (Fig. 3b). The identification of one diploid clone indicated that the original BANAN/83/CRTRA/64 parasite population was in fact a mixed infection between triploid and diploid parasites. We tried to estimate the proportion of BANANCL1 in BANAN/83/CRTRA/64 based on specific homozygous SNP markers. However, at each individual SNP level, BANAN/83/CRTRA/64 and

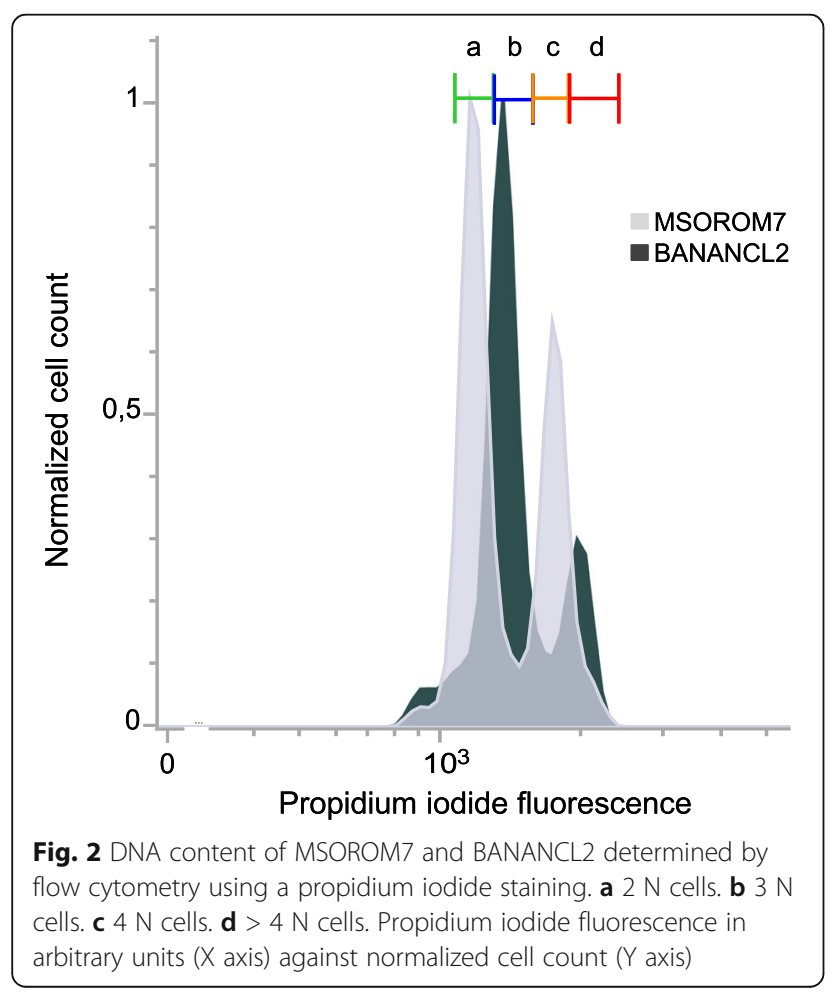

BANANCL2 were very similar and there were no BANANCL1 specific homozygous SNP markers that separated BANANCL1 from BANAN/83/CRTRA/64 and BANANCL2. Therefore, the proportion of BANANCL1 in the BANAN/83/CRTRA/64 sample was undetectably low, suggesting that BANANCL1 was not a dominant population in the BANAN/83/CRTRA/64 isolate. In addition, the similarity between the block alternative allele frequency profiles of BANAN/83/CRTRA/

Table 1 Sequencing data. Read depth information of BANANCL2 life-cycle stages mapped to the T. congolense IL3000 reference genome following WGS. The parasites were collected from the tsetse midgut (MG), proventriculus (PV), proboscis (PB) and from the infected mouse after the tsetse fly bite (MOU)

\begin{tabular}{lllll}
\hline & Average depth & \multicolumn{2}{l}{ Total reads } & \multicolumn{2}{c}{ Mapped reads } \\
\cline { 4 - 6 } & & $(\mathrm{M})$ & $(\mathrm{M})$ & $(\%)$ \\
\hline BANAN/83/CRTRA/64 & 19.2 & 2.61 & 2.51 & 96.0 \\
BANAN-MG-1 & 3.5 & 2.21 & 0.39 & 17.7 \\
BANAN-MG-2 & 7.4 & 3.35 & 0.86 & 25.5 \\
BANAN-MG-3 & 3.9 & 3.65 & 0.47 & 12.9 \\
BANAN-PB-1 & 1.9 & 3.62 & 0.23 & 6.4 \\
BANAN-PB-2 & 1.7 & 3.39 & 0.13 & 3.9 \\
BANAN-PB-3 & 2.7 & 3.07 & 0.30 & 9.9 \\
BANAN-PV-1 & 3.3 & 3.04 & 0.41 & 13.6 \\
BANAN-PV-2 & 2.6 & 2.76 & 0.26 & 9.6 \\
BANAN-PV-3 & 19.2 & 3.79 & 2.13 & 56.1 \\
BANAN-MOU-1 & 36.2 & 4.42 & 3.69 & 83.4 \\
BANAN-MOU-2 & 31.5 & 3.86 & 3.21 & 83.2 \\
BANAN-MOU-3 & 35.1 & 4.65 & 3.78 & 81.3 \\
BANANCL1 & 8.9 & 1.70 & 1.60 & 93.7 \\
BANANCL2 & 79.0 & 11.45 & 10.92 & 95.4 \\
BANANCL3 & 24.6 & 4.23 & 3.98 & 94.0 \\
\hline M million & & & &
\end{tabular}




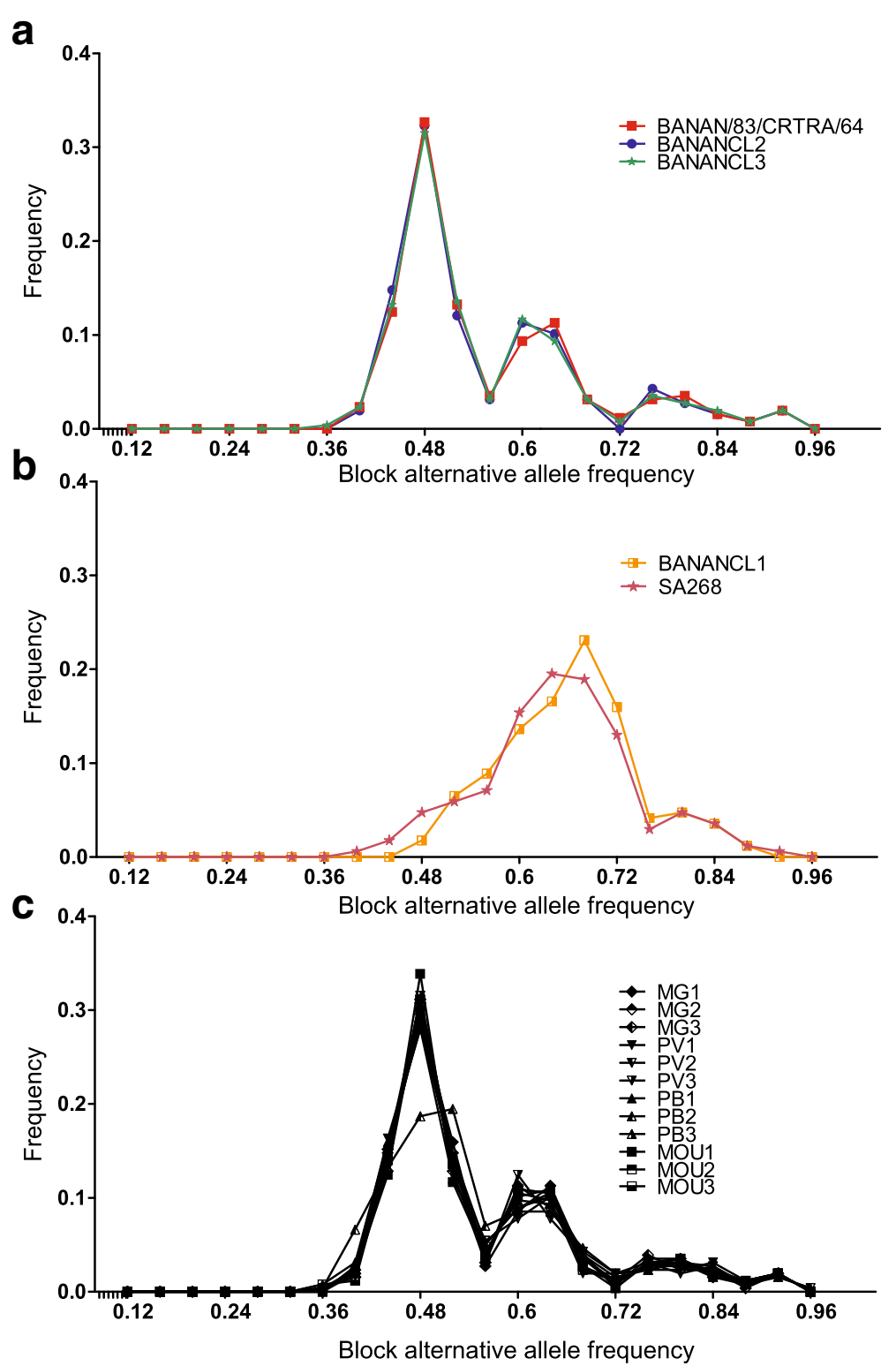

Fig. 3 Block alternative allele frequency profiles. The block alternative allele frequency profiles based on windows of 1000 SNPs are shown for all the different BANAN samples. This technique enabled the estimation of the alternate allele frequencies in samples with low read depth. a The block frequency profile of BANAN/83/CRTRA/64, BANANCL3 and BANANCL2. $\mathbf{b}$ The block frequency profile of the diploid Burkinabe sample $T$. congolense SA268 is shown here as diploid control. c All the BANANCL2 samples showed a frequency profile characteristic of triploid samples (see main text and Methods). In contrast, BANANCL1 showed an alternate allele frequency profile with one peak characteristic to diploid samples. Abbreviations: MG, midgut; PV, proventriculus; PB, proboscis; MOU, mouse

64 and BANANCL2 (i.e. correlation value of $r^{2}=0.996$ ) also indicated that the triploid population was the predominant population found in this isolate.

\section{Maintenance of triploidy throughout the $T$. congolense life-cycle}

We next determined the ploidy status of the BANANCL2 strain throughout its life-cycle after infections of G. morsitans morsitans tsetse flies (i.e. three groups of independently infected flies). At 28 days postinfection, proboscis-positive tsetse flies were detected in all three groups. The bite of these infected flies successfully resulted in a systemic infection of mice. Parasites were isolated for WGS analyses from the midgut, proventriculus and proboscis of the flies, and from the blood of infected mice. These isolates represented the main life-cycle stages of $T$. congolense. Paired-end reads were mapped against the reference T. congolense IL3000 
Table 2 Correlation values between the block alternative allele frequencies of the parasites in the three mouse groups and their respective parasite stages in the different tsetse fly tissues

\begin{tabular}{lllll}
\hline ID & ID & $r$ & $r^{2 a}$ & $P$-value \\
\hline BANAN/83/CRTRA/64 & BANANCL2 & 0.998 & 0.996 & $<0.0001$ \\
BANANCL1 & BANANCL2 & 0.585 & 0.342 & $<0.0001$ \\
BANANCL3 & BANANCL2 & 0.998 & 0.997 & $<0.0001$ \\
BANAN-MOU-1 & BANAN-MG-1 & 0.994 & 0.988 & $<0.0001$ \\
BANAN-MOU-1 & BANAN-PB-1 & 0.988 & 0.975 & $<0.0001$ \\
BANAN-MOU-1 & BANAN-PV-1 & 0.991 & 0.981 & $<0.0001$ \\
BANAN-MOU-2 & BANAN-MG-2 & 0.996 & 0.992 & $<0.0001$ \\
BANAN-MOU-2 & BANAN-PB-2 & 0.969 & 0.938 & $<0.0001$ \\
BANAN-MOU-2 & BANAN-PV-2 & 0.989 & 0.978 & $<0.0001$ \\
BANAN-MOU-3 & BANAN-MG-3 & 0.992 & 0.983 & $<0.0001$ \\
BANAN-MOU-3 & BANAN-PB-3 & 0.991 & 0.982 & $<0.0001$ \\
BANAN-MOU-3 & BANAN-PV-3 & 0.997 & 0.995 & $<0.0001$
\end{tabular}

${ }^{a}$ The coefficients of determination $r^{2}$ are high between BANANCL2, BANAN/83/ CRTRA/64 and BANANCL3, as well as between the tsetse-bite infecting mice samples and the tsetse samples, indicating that the overall allele frequencies remained triploid in all these groups. In contrast, the coefficient of determination $r^{2}$ is low between BANANCL1 and BANANCL2, indicative of their genomic differences

${ }^{\mathrm{b}}$ The $P$-values were calculated by a two-tailed test

Abbreviations: $M G$ midgut, $P V$ proventriculus, $P B$ proboscis, $M O U$ mouse

genome [18]. The average depth coverage was $4.9 \times, 8.4 \times$ and $2.1 \times$ for parasites extracted from the tsetse fly midgut, proventriculus and proboscis, respectively (Table 1). The average depth coverage was $34.3 \times$ for parasites isolated from the murine hosts. The depth coverage of parasites isolated from flies was low, and on average only $17.3 \%$ of the reads could be mapped to the $T$. congolense reference genome because of the difficulty in extracting pure parasite fractions without any tsetse tissue contaminations.

Despite the lower read depth for these samples, we were able to estimate the ploidy of parasite populations isolated from the different tsetse fly tissues, and from the blood of the infected mice, using the block alternative allele frequency method. We observed that the alternative allele frequency profiles were all similar to the frequency profile of the triploid sample BANANCL2 (Fig. 3c), except for one proboscis-derived parasite (BANAN-PB-2) which had a very low depth coverage (i.e. $1.7 \times$ coverage; Table 1 ). Nevertheless, we identified that this profile corresponded to a triploid sample with lower depth by using additional verifications (see Methods). The read depths of the blood stream parasites extracted from the infected mice were $>30 \times$ (Table 1 ). We were therefore able to identify their triploid patterns from individual chromosomes using the alternative allele frequency spectra based on individual SNP sites and observed two distinct peaks around 0.33 and 0.66 (Fig. 1c).
To provide statistical significance of the outcome of the block alternative allele frequency method for the low read depth samples, we calculated the coefficient of determination $\left(r^{2}\right)$ between the block alternative allele frequencies of the parasites in the three mice groups (i.e. we confirmed triploidy based on the alternative allele frequency profiles, Fig. 1c) and their respective parasite stages in the different tsetse fly tissues. All $r^{2}$ values were found to be high, indicating that the overall allele frequencies remained triploid in all parasite stages (Table 2 ). In contrast, the $r^{2}$ values between the block alternative allele frequencies of the diploid strain BANANCL1 and the triploid strain BANANCL2 was low, reflecting the genetic differences between both strains (Table 2).

Finally, we analyzed the genome sequence data from the different BANANCL2 parasite life-cycle stages in order to look for genetic signatures of recombination in the parasites, such as long losses of heterozygous sites or long traces of shifts in SNP heterozygosity, as previously observed in WGS analyses [11, 19]. The presence of such genetic signatures at a specific $T$. congolense lifecycle stage would be indicative of an elevated recombination activity at that stage and could in turn reflect a potential site of parasite sexual exchange where triploid cells can emerge accidentally. However, we found no evidence of large genomic changes and did not observe genomic footprints of genetic recombination.

\section{Discussion}

The genome of African trypanosomes is reported to be diploid [1, 2]. However, our results demonstrated that triploid $T$. congolense parasites can complete a full lifecycle, and that triploid populations in the field are viable and sustained in host populations. We used a WGS approach to estimate the state of ploidy of $T$. congolense populations during each stage of the parasite life-cycle. We then developed a method based on the block alternative allele frequency to accurately detect the ploidy profiles of samples with low sequencing depth. This method could be extended to other organisms and low parasitemia samples including those directly collected in the field for ploidy estimation.

When analyzing the ploidy status of the field isolate BANAN/83/CRTRA/64 and its clones BANANCL1, BANANCL2 and BANANCL3, we found that BANAN/ 83/CRTRA/64 was a mixed infection between diploid and triploid populations, with the triploid population predominating. From this, we hypothesize that the triploidy that emerged in this BANAN natural population conferred a beneficial advantage to the diploid counterpart in their local ecological/epidemiological setting.

The advantages and disadvantages of polyploidy have been reported in various organisms. For instance, advantages associated with changes in ploidy have been 
described in Leishmania parasites [20, 21], in T. cruzi parasites [22, 23], in yeasts [24], and in the fungal pathogen Cryptococcus neoformans [25, 26]. In Leishmania major, changes in the number of particular chromosomes in the genome (i.e. aneuploidy) resulted in gene amplifications and were associated with drug resistance $[20,21]$. In yeast, whole-genome duplication (i.e. polyploidy) has been shown to play a key role in their evolutionary adaptation to stressful conditions [24]. Polyploidy increased the genetic diversity of the yeast population contributing to the acquisition and spread of beneficial mutations. Similarly in C. neoformans, rates of survival and reproduction of polyploid cells were better under stressful conditions in comparison to their haploid counterparts [25].

The increase in ploidy enlarged the size of the genome, enhancing the content and diversity of the gene pool. Some of the newly acquired genes might confer phenotypic advantages to the strain, such as resistance to a drug, adaptation to stressful conditions, or changes in virulence and pathogenicity.

Triploidy could also be associated with reduced viability and reduced chances for a strain to complete a full life-cycle because of replication costs associated with the increased DNA size of the genome [5]. However, this does not seem to be the case here since the triploid population in the original field isolate surpassed the diploid population, suggesting that its increased DNA size did not affect its capability to establish a systemic infection.

Since we have isolated one triploid sample out of $56 \mathrm{~T}$. congolense parasites sequenced [11], we suspect that triploidy may play a larger role in $T$. congolense natural populations as currently assumed. The emergence of triploid and other polyploid trypanosome cells during experimental crossings between $T$. brucei parasites is not uncommon [5], and it can be assumed that a similar scenario is also happening in $T$. congolense. However, whether our observation of triploidy in a natural T. congolense population is an isolated case or occurs more frequently remains to be verified.

To date, the frequency of triploid $T$. congolense parasites in natural populations is unknown partly because few studies have been designed for its identification. For instance, identifying triploidy with routine approaches, such as microsatellites genotyping, is not practical since a triploid profile is nearly identical to profiles of mixed infections. However, the emergence of Next Generation Sequencing technologies will greatly improve the detection of these parasite populations in future, and will provide further information about their frequency.

Triploid cells could emerge due to errors during meiosis. We previously demonstrated the presence of recombination breakpoints in the genome of the original
BANANCL2 line [11]. Here, we analyzed whole genome sequence data extracted from BANANCL2 at each lifecycle stage to identify recombination signatures and determine the life-cycle stage at which recombination, and therefore a potential emergence of triploidy, is taking place in T. congolense. However, there was no genomic evidences for new recombination events in our collection of samples, partly because recombination between clonal cells is difficult to detect. It would be informative to further explore meiosis in $T$. congolense and perform experimental crossings as described in T. brucei [5] to further address the potential link between meiosis and triploidy.

In addition to failures during meiosis, the triploid parasite population could also have emerged due to errors during mitosis (reviewed in: [27]). Here, errors at the different checkpoints of mitotic division could have led to improper chromosome segregation and the emergence of daughter cells with improper number of chromosomes.

\section{Conclusion}

Our finding is a recent addition to increasing observations of changes in ploidy found in trypanosomatid parasites such as clinical and laboratory lines of Leishmania spp. [17, 21, 28, 29] and T. cruzi parasites [22, 23], highlighting its prevalence and significance in kinetoplastids. The triploid parasites identified in this study represent an alternative model system to study different aspects of the $T$. congolense biology, such as gene expression levels related to recombination, drug resistance and other stress responses. In addition, comparing triploid to diploid parasites can provide unique insights into the impact of chromosome copy number variations in $T$. congolense natural populations.

\section{Additional file}

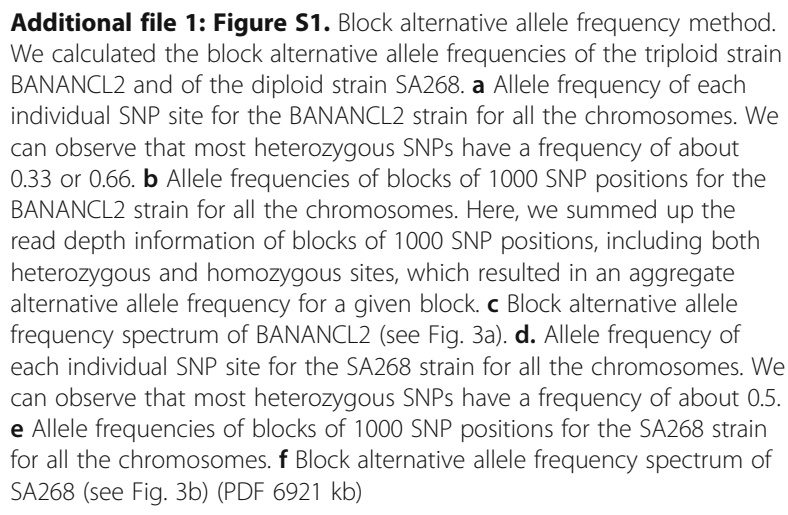

Additional file 1: Figure S1. Block alternative allele frequency method. We calculated the block alternative allele frequencies of the triploid strain BANANCL2 and of the diploid strain SA268. a Allele frequency of each individual SNP site for the BANANCL2 strain for all the chromosomes. We can observe that most heterozygous SNPs have a frequency of about 0.33 or 0.66 . $\mathbf{b}$ Allele frequencies of blocks of 1000 SNP positions for the BANANCL2 strain for all the chromosomes. Here, we summed up the read depth information of blocks of 1000 SNP positions, including both heterozygous and homozygous sites, which resulted in an aggregate alternative allele frequency for a given block. c Block alternative allele frequency spectrum of BANANCL2 (see Fig. 3a). d. Allele frequency of each individual SNP site for the SA268 strain for all the chromosomes. We can observe that most heterozygous SNPs have a frequency of about 0.5 . e Allele frequencies of blocks of 1000 SNP positions for the SA268 strain for all the chromosomes. $\mathbf{f}$ Block alternative allele frequency spectrum of SA268 (see Fig. 3b) (PDF 6921 kb)

\section{Abbreviations}

MG: Midgut; MOU: Mouse; PB: Proboscis; PV: Proventriculus; WGS: Whole Genome Sequencing 


\section{Acknowledgements}

We thank Lieve Vermeiren for helping with the sequencing of the samples. We thank Adam Hendy for proofreading the article.

\section{Funding}

This work was financed by the EU-GPARD, Grant reference number: TRYRAC/ DCI-FOOD/2011/279-754, and the Department of Economy, Science and Innovation in Flanders, ITM-SOFIB GemiNI.

\section{Availability of data and materials}

The dataset generated and analyzed during the current study is available in EBI under the accession number PRJEB21681 (http://www.ebi.ac.uk/ena/data/ view/PRJEB21681)

\section{Authors' contributions}

ET: conception and design, acquisition of data, completed experimental analysis, performed additional data analysis and visualization, analysis and interpretation of data, drafting or revising the article. HI: performed SNP detection, aligned reads and determined read depth, developed the block alternative allele frequency method, performed additional data analysis and visualization, analysis and interpretation of data, drafting or revising the article. JCD: conception and design, interpretation of data, drafting or revising the article. JVDA: conception and design, interpretation of data, drafting or revising the article. All authors read and approved the final manuscript.

\section{Ethics approval and consent to participate}

The experimental animal work was approved by the Institute of Tropical Medicine (ITM) animal ethics committee, clearance numbers DG020 and VP2014-2.

\section{Consent for publication}

Not applicable.

\section{Competing interests}

The authors declare that they have no competing interests.

\section{Publisher's Note}

Springer Nature remains neutral with regard to jurisdictional claims in published maps and institutional affiliations.

Received: 11 July 2017 Accepted: 26 September 2017 Published online: 10 October 2017

\section{References}

1. Borst P, van der Ploeg M, van Hoek JF, Tas J, James J. On the DNA content and ploidy of trypanosomes. Mol Biochem Parasitol. 1982;6:13-23.

2. Gibson WC, Osinga KA, Michels PA, Borst P. Trypanosomes of subgenus Trypanozoon are diploid for housekeeping genes. Mol Biochem Parasitol. 1985;16(3):231-42.

3. Hope M, MacLeod A, Leech V, Melville S, Sasse J, Tait A, Turner CM. Analysis of ploidy (in megabase chromosomes) in Trypanosoma brucei after genetic exchange. Mol Biochem Parasitol. 1999;104(1):1-9.

4. Gibson W, Garside L, Bailey M. Trisomy and chromosome size changes in hybrid trypanosomes from a genetic cross between Trypanosoma brucei rhodesiense and T. b. brucei. Mol Biochem Parasitol. 1992;51 (2):189-99.

5. Gibson W, Peacock L, Ferris V, Williams K, Bailey M. The use of yellow fluorescent hybrids to indicate mating in Trypanosoma brucei. Parasit Vectors. 2008; 1(1):4.

6. MacLeod A, Turner CMR, Tait A. Chapter 3: The system of genetic exchange in Trypanosoma brucei and other trypanosomatids. In: Barry JD, McCulloh R, Mottram JC, Acosta-Serrano A, editors. . Glasgow: Horizon Press; 2007. p. 2007.

7. Gibson W, Stevens J. Genetic exchange in the trypanosomatidae. Adv Parasitol. 1999:43:1-46.

8. Peacock L, Ferris V, Bailey M, Gibson W. Mating compatibility in the parasitic protist Trypanosoma brucei. Parasit Vectors. 2014;7:78

9. Peacock L, Ferris V, Sharma R, Sunter J, Bailey M, Carrington M, Gibson W. Identification of the meiotic life-cycle stage of Trypanosoma brucei in the tsetse fly. Proc Natl Acad Sci USA. 2011;108(9):3671-6.

10. Peacock L, Bailey M, Carrington M, Gibson W. Meiosis and haploid gametes in the pathogen Trypanosoma brucei. Curr Biol. 2014;24(2):181-6.
11. Tihon E, Imamura H, Dujardin JC, Van Den Abbeele J, Van den Broeck F. Discovery and genomic analyses of hybridization between divergent lineages of Trypanosoma congolense, causative agent of animal African trypanosomiasis. Mol Ecol. 2017; 10.1111/mec.14271.

12. Pinder $M$, Authie $E$. The appearance of isometamidium resistant Trypanosoma congolense in West Africa. Acta Trop. 1984;41(3):247-52.

13. Masumu J, Geysen D, Van den Bossche P. Endemic type of animal trypanosomiasis is not associated with lower genotype variability of Trypanosoma congolense isolates circulating in livestock. Res Vet Sci. 2009;87(2):265-9.

14. Clausen PH, Sidibe I, Kabore I, Bauer B. Development of multiple drug resistance of Trypanosoma congolense in Zebu cattle under high natural tsetse fly challenge in the pastoral zone of Samorogouan, Burkina Faso. Acta Trop. 1992:51(3-4):229-36.

15. Elsen P, Van Hees J, De Lil EL. historique et les conditions d'élevage des lignées de glossines (Diptera, Glossinidae) maintenues à l'institut de Médecine tropicale Prince Léopold d'Anvers. Rev Zool Afr. 1993;107:439-49.

16. Caljon G, Van Reet N, De Trez C, Vermeersch M, Perez-Morga D, Van Den Abbeele J. The dermis as a delivery site of Trypanosoma brucei for tsetse flies. PLoS Pathog. 2016;12(7):e1005744.

17. Rogers MB, Hilley JD, Dickens NJ, Wilkes J, Bates PA, Depledge DP, et al. Chromosome and gene copy number variation allow major structural change between species and strains of Leishmania. Genome Res. 2011; 21(12):2129-42.

18. Jackson AP, Berry A, Aslett M, Allison HC, Burton P, Vavrova-Anderson J, et al. Antigenic diversity is generated by distinct evolutionary mechanisms in African trypanosome species. Proc Natl Acad Sci USA. 2012:109(9):3416-21.

19. Rogers MB, Downing T, Smith BA, Imamura H, Sanders M, Svobodova M, et al. Genomic confirmation of hybridisation and recent inbreeding in a vector-isolated Leishmania population. PLoS Genet. 2014;10(1):e1004092.

20. Martinez-Calvillo S, Stuart K, Myler PJ. Ploidy changes associated with disruption of two adjacent genes on Leishmania major chromosome 1. Int J Parasitol. 2005;35(4):419-29.

21. Ubeda JM, Legare D, Raymond F, Ouameur AA, Boisvert S, Rigault P, et al. Modulation of gene expression in drug resistant Leishmania is associated with gene amplification, gene deletion and chromosome aneuploidy. Genome Biol. 2008;9(7):R115.

22. Souza RT, Lima FM, Barros RM, Cortez DR, Santos MF, Cordero EM, et al. Genome size, karyotype polymorphism and chromosomal evolution in Trypanosoma cruzi. PLoS One. 2011;6(8):e23042.

23. Lima FM, Souza RT, Santori FR, Santos MF, Cortez DR, Barros RM, et al. Interclonal variations in the molecular karyotype of Trypanosoma cruzi: chromosome rearrangements in a single cell-derived clone of the $\mathrm{G}$ strain PLoS One. 2013;8(5):e63738.

24. Selmecki AM, Maruvka YE, Richmond PA, Guillet M, Shoresh N, Sorenson AL, et al. Polyploidy can drive rapid adaptation in yeast. Nature. 2015;519(7543):349-52.

25. Gerstein AC, MS F, Mukaremera L, Li Z, Ormerod KL, Fraser JA, et al. Polyploid titan cells produce haploid and aneuploid progeny to promote stress adaptation. MBio. 2015;6(5):e01340-15.

26. Bennett RJ, Forche A, Berman J: Rapid mechanisms for generating genome diversity: whole ploidy shifts, aneuploidy, and loss of heterozygosity. Cold Spring Harb Perspect Med 2014;4(10) pii: a019604.

27. Potapova T, Gorbsky GJ. The consequences of chromosome segregation errors in mitosis and meiosis. Biology (Basel). 2017;6(1).

28. Downing T, Imamura H, Decuypere $\mathrm{S}$, Clark TG, Coombs GH, Cotton JA, et al. Whole genome sequencing of multiple Leishmania donovani clinical isolates provides insights into population structure and mechanisms of drug resistance. Genome Res. 2011;21(12):2143-56.

29. Leprohon P, Legare D, Raymond F, Madore E, Hardiman G, Corbeil J, Ouellette M. Gene expression modulation is associated with gene amplification, supernumerary chromosomes and chromosome loss in antimony-resistant Leishmania infantum. Nucl Acids Res. 2009;37(5):1387-99. 